whether animal-based or not, unless they are entirely satisfied that the alternative is valid, meets the requirements laid down in national and international guidelines for validity (International Conference on Harmonisation, 2005; United States Pharmacopeia, 2010), and correlates/is comparable with the current $\mathrm{LD}_{50}$ method. These requirements generally are different from those applied to other animal assay replacements (Worth and Balls, 2002). The mouse $\mathrm{LD}_{50}$ assay is the "gold standard" for any potency measurement of BoNT, and to dethrone such a standard requires an approach that is both scientifically sound and relevant to the specific product being tested.

The mouse $\mathrm{LD}_{50}$ assay, as validated by all the manufacturers, measures all four properties of BoNT; binding to the neuromuscular junction (NMJ) receptors, endocytotic internalization into the NMJ cytosol, translocation of the active portion of the BoNT molecule (the Light Chain), and final enzymatic cleavage of the relevant target substrate. Replication of each of these properties individually is possible but in vitro combinations of even two are rarely achieved and scientifically challenging. Ex vivo models, using isolated animal organs, are available which can replicate the $\mathrm{LD}_{50}$ but are generally impractical to apply routinely and/or require significant numbers of organs to achieve appropriate results. In other words, the current state of BoNT science does not permit a direct replacement at present without the use of animals.

Contrary to statements that have often been made in various publications and commentaries, the SNAP-25 endopeptidase assay method, developed by the UK National Institute for Biological Standards and Control (NIBSC) for specific internal use, has never been accepted for product quality testing and release by any worldwide regulatory authority, since it only measures one property of BoNT. Although used by and adequately established for their own purposes, this method has also not been validated in accordance with the requirements of the European Pharmacopoeia, regulatory authorities worldwide or the $\mathrm{ICH}$ guidelines. Additionally, transfer of this assay method outside of NIBSC would be required in order to demonstrate its validity (inter-laboratory testing) (Sesardic, 2010). Until such data are available and assembled for review by the competent authorities, the assay will remain an internal method only.

All parties involved, manufacturers, scientists and regulators, share Dr. Bitz' desire for an alternative to the $\mathrm{LD}_{50}$ potency assay, and are working continuously with this aim, as reported in detail (Adler et al., 2010). Several potential alternative assays have been comprehensively studied and have not met the regulatory criteria. Others are currently under study, and BoNT science continues to deliver new prospects for investigation, despite commentaries to the contrary.

\section{References}

Adler, S., Bicker, G., Bigalke, H. et al. (2010). The current scientific and legal status of alternative methods to the $\mathrm{LD}_{50}$ test for botulinum neurotoxin potency testing. ATLA 38, 1-15.

Bitz, S. (2010). The botulinum neurotoxin $\mathrm{LD}_{50}$ test - problems and solutions. ALTEX 27, 114-116.

International Conference on Harmonisation (2005). Validation of analytical procedures: Text and methodology Q2R(1).

Sesardic, D. (2010). Is it possible to accurately determine content of botulinum neurotoxin type A in drug products? Drugs in $R \& D 10,91-92$.

Straughan, D. W. (2006). Progress in applying the three Rs to the potency testing of botulinum toxin type A. ATLA 34(3), 305-313.

United States Pharmacopoeia (2010). General chapter biological assay validation $<1033>$.

Worth, A. P. and Balls, M. (ed.) (2002). Alternative (non-animal) methods for chemicals testing: Current status and future prospects. Chapter 2. The principles and procedures of validation. ATLA 30, Suppl. 1, 13-19.

\title{
Reply to comment on "The Botulinum Neurotoxin LD 50 Test - Problems and Solutions"
}

\author{
Silke Bitz \\ Doctors Against Animal Experiments Germany, Munich, Germany \\ e-mail: info@aerzte-gegen-tierversuche.de
}

Dr. Pickett dismisses the cosmetic use of botulinum toxin products out of hand. He criticises "...the incorrect statement that the toxin products are used as cosmetics, accusing the manufacturers of exploiting legal loopholes that permit the testing of cosmetics on animals. BoNT is not a cosmetic product." Indeed, although used to a high percentage for cosmetic purposes, botulinum toxin (BoNT) products are defined as pharmaceuticals, because they are injected into the body, as explained in the original article (Bitz, 2010). Thus pharmaceutical safety testing is required by law even though the application is often aesthetic.
To comply with the $7^{\text {th }}$ Amendment of the Cosmetics Directive, which bans animal testing for cosmetic products and ingredients, politics is obliged to close this gap to prevent animal tests for products that are used extensively for aesthetic reasons.

It is impossible to deny the fact that these products are being used not only for medical but also largely for cosmetic applications. Product names such as Botox ${ }^{\circledR}$ Cosmetic suggest that BoNT is used not solely for medical purposes. Also, information published by the manufacturers themselves confirms its use for cosmetic purposes. For example, Allergan in its Annual Report 
2008 states: "The net sales growth of 12 percent we achieved in 2008 was quite different from our expectations at the start of the year, particularly with regard to continued strong expansions of the medical aesthetics business that includes Botox ${ }^{\circledR}$ Cosmetic, dermal filler and breast aesthetics." In the same report it is pointed out that the partnership with GlaxoSmithKline enabled Japanese approval of Botox ${ }^{\circledR}$ for aesthetic use in 2009. Further, Ipsen's Annual Report 2008 informs “ “...the FDA granted marketing authorisation for Dysport ${ }^{\mathrm{TM}}$... for the treatment of cervical dystonia and also for aesthetic medicine." Further the report states, "Azzalure ${ }^{\circledR}$ received the collective green light ... in aesthetic use for the treatment of frown lines." It is further stated that Dysport ${ }^{\circledR}$ is marketed for aesthetic indications by Medics in the US and in Europe by Galderma, under the Azzalure ${ }^{\circledR}$ brand, and that the group has granted Galderma the exclusive right to develop, promote, and distribute its botulinum toxin type A for aesthetic indications in Europe and certain other territories (Ipsen, 2009).

According to the manufacturers themselves, cosmetic uses account for around one half of all applications. Allergan stated in 2005, "Presently, Botox ${ }^{\circledR}$ is approved for 20 indications in more than 75 countries, with an estimated 57 percent of sales relating to therapeutic uses and 43 percent to aesthetic use" (Allergan, 2005).

The "off-label"use of BoNT products cannot be denied. In Switzerland, for example, it is en vogue for many people to use their lunch hour to visit a beauty clinic to get a beauty treatment with a botulinum toxin product. It is highly doubtful that severe psychological reasons cause people to visit a beauty clinic. Therefore, it is incorrect of Dr. Pickett to claim that botulinum toxin is applied solely for medical indications.

However, independent of whether BoNT products are used for cosmetic or medical indications, it is unsustainable that the manufacturers are not committed strongly enough to support the deletion of the $\mathrm{LD}_{50}$ test from the European Pharmacopoeia by validating one of the available and allowed alternative tests. For the animals used in the cruel and scientifically unreliable $\mathrm{LD}_{50}$ test, it does not matter whether the product tested on them will be used for medical or for aesthetic reasons. The suffering experienced by the mice is always the same: they die over the course of a couple of days by asphyxiation. Additionally, extremely cruel procedures have been filmed undercover in Ipsen's contract laboratory in Wickham, Hampshire (http://www.buav.org/ investigations/theuglytruthaboutbotox).

Dr. Pickett claims that he wants to give an accurate update on the current situation and is of the opinion that the estimated number of animals used for BoNT testing is incorrect. However, in his comment any figure, number, or detail on animal use which might prove estimated animal numbers wrong is completely missing. Only the manufacturers know the exact numbers of animals who die in the $\mathrm{LD}_{50}$ test for BoNT products. Ipsen and the other manufacturers of BoNT products should be made to share their complete data on animal experimentation with the public and to publish their exact records on how many animals undergo testing of BoNT products annually. Of course, in order to have access to the most accurate state of affairs, the manufacturers also have to be open to independent audits at any time without notice.

In his statement, Dr. Pickett further appeals to the fact that the $\mathrm{LD}_{50}$ test is regarded as the "gold standard." However, it is a matter of fact that the $\mathrm{LD}_{50}$ test is criticised by scientists, not only because of its cruelty, but also for its lack of scientific reliability (Hartung, 2009). Further, this animal-based assay has never been validated and is thus regarded unproven as "gold standard."

The European Pharmacopoeia clearly allows three alternatives which are preferable to the conventional mouse assay in terms of animal welfare subject to validation (European Pharmacopoeia, 2006). Dr. Pickett states, that the Snap-25 endopeptidase assay, which is one of the allowed alternatives developed by the National Institute for Biological Standards and Controls in the United Kingdom, “... has never been accepted for product quality testing and release by any worldwide regulatory authority." Here, Ipsen and the other manufacturers of BoNT products are asked to submit data on what exactly they have undertaken to validate this test or any other alternative test for their products and to make their validation study, aiming at the regulatory acceptance of the Snap 25-assay or another test, publicly available.

Apart from the severe animal welfare problem BoNT testing is causing, it is irresponsible of the manufacturers to also accept that people are subjected to a risk. Several adverse effects and deaths due to BoNT products have been reported (FDA, 2008; arznei-telegramm, 2007). This shows that the $\mathrm{LD}_{50}$ test is not able to ensure safety for patients or persons undergoing BoNT treatments for aesthetic reasons.

As long as the manufacturers are not willing to intensively promote the deletion of the $\mathrm{LD}_{50}$ test on mice, a ban on the use of BoNT products for cosmetic use is urgently needed while on a scientific and political level the mandatory application of an animal-free method for BoNT testing must be driven forward.

\section{References}

Allergan (2005). Annual Report.

Allergan (2008). Annual Report.

arznei-telegramm (2007). 38 (9), 88. http://www.arzneimitteltelegramm.de/zeit/0709_a.php3.

Bitz, S. (2010). The botulinum neurotoxin $\mathrm{LD}_{50}$ test - problems and solutions. ALTEX 27, 114-116.

European Pharmacopoeia (2006). Botulinum toxin Type A for injection, 01/2005, 2113. In European Pharmacopoeia 6.0, 1117-1119.

FDA (2008). Early communication about an ongoing safety review of botox and botox cosmetic (Botulinum toxin Type A) and myobloc (Botulinum toxin Type B). http://www.fda.gov/ Drugs/DrugSafety/PostmarketDrugSafetyInformationforPatientsandProviders/DrugSafetyInformationforHeathcareProfessionals/ucm070366.htm

Hartung, T. (2009). Toxicology for the twenty-first century. $\mathrm{Na}$ ture 460, 208-212.

Ipsen (2008). Annual Report.

Ipsen (2009). Annual Report. 